\title{
Skepticism and rumor spreading: The role of spatial correlations
}

\author{
Marco Antonio Amaral $\odot,{ }^{1, *}$ W. G. Dantas $\odot,{ }^{2,3, \dagger}$ and Jeferson J. Arenzon $\oplus^{3,4, \$}$ \\ ${ }^{1}$ Instituto de Humanidades, Artes e Ciências, Universidade Federal do Sul da Bahia, CEP 45638-000 Teixeira de Freitas, Bahia, Brazil \\ ${ }^{2}$ Departamento de Ciências Exatas, EEIMVR, Universidade Federal Fluminense, CEP 27255-125, Volta Redonda, Rio de Janeiro, Brazil \\ ${ }^{3}$ Instituto de Física, Universidade Federal do Rio Grande do Sul, CEP 91501-970 Porto Alegre, Rio Grande do Sul, Brazil \\ ${ }^{4}$ Instituto Nacional de Ciência e Tecnologia - Sistemas Complexos, Rio de Janeiro, Rio de Janeiro, Brazil
}

(Received 1 April 2020; accepted 5 June 2020; published 25 June 2020)

\begin{abstract}
Critical thinking and skepticism are fundamental mechanisms that one may use to prevent the spreading of rumors, fake news, and misinformation. We consider a simple model in which agents without previous contact with the rumor, being skeptically oriented, may convince spreaders to stop their activity or, once exposed to the rumor, decide not to propagate it as a consequence, for example, of fact checking. We extend a previous, mean-field analysis of the combined effect of these two mechanisms, active and passive skepticism, to include spatial correlations. This can be done either analytically, through the pair approximation, or simulating an agent-based version on diverse networks. Our results show that while in mean field there is no coexistence between spreaders and susceptibles (although, depending on the parameters, there may be bistability depending on the initial conditions), when spatial correlations are included, because of the protective effect of the isolation provided by removed agents, coexistence is possible.
\end{abstract}

DOI: 10.1103/PhysRevE.101.062418

\section{INTRODUCTION}

Polarization and fake-news propagation are intertwined social phenomena that may be amplified by a systemic lack of critical thinking and skeptical skills among the population [1-4]. Rumor- or opinion-spreading models [5,6] provide simple descriptions of how misinformation and gossips diffuse between networked individuals, in a similar way as contagious diseases propagate through direct contact [7-10]. In the latter, where the contamination is usually involuntary and unconscious, a deeper understanding of the process has led to a more effective protection of susceptible populations through better designed vaccination [11-17], isolation [18,19], and quarantine [20-22] campaigns. The comprehension of the main underlying mechanisms in the case of rumor propagation, involving an intentional and directed action from the spreader, may help to devise efficient strategies to counteract the effects of anti- and pseudoscience movements. Similar programs have been proposed, for example, to confront Internet hate speech [23], organized crime [24], and terrorism [25,26].

Many analogies have been drawn between the processes of rumor and disease propagation through contact. The simplest models for the above contact processes [27] explore their similarity and consider three dynamical states: ignorants, spreaders, and stiflers [28-32] for rumor propagation, and susceptible, infected, and removed (or recovered) [33] for diseases. While unaware of the rumor or uncontaminated by the disease, the agent is susceptible $(S)$ to it. After being exposed, if the agent does not become a spreader (state $Z$ ), it turns into

\footnotetext{
*marcoantonio.amaral@cpf.ufsb.edu.br

†wgdantas@id.uff.br

†arenzon@ufrgs.br
}

a stifler (for example, resisting and getting removed from the propagation pool, state $R$ ). More refined models introduced further states and mechanisms [6,8,34]. For example, there is an intermediate, exposed state- that is relevant for the model introduced here-corresponding to a noncontagious period after the contact with a spreader $[35,36]$, when the agent hesitates between becoming $Z$ or $R$.

Fake news, misinformation, hoaxes, and pseudoscience propagate among the population through least-resistance paths, getting reinforced by new technologies, and are rarely completely removed. Several works have tried to measure, identify, and model their mechanisms and effects $[37,38]$. An essential ingredient is the presence of skeptical agents. In standard disease or rumor propagation, only the susceptible is affected by its interaction with a spreader. Skepticism may, instead, not only prevent the susceptible to become infected but also (i) change the spreader, removing it from the propagation pool, and (ii) prevent an already exposed agent to become a spreader. While an active skeptical susceptible may convince its neighbor spreader, the convincing process of an exposed individual is passive (by consulting the original sources and the available literature, for example). In a previous work [39], a simple model introduced the opposing behavior of skeptical agents and was studied within the one-site mean-field approximation. However, the effect of spatial correlations was not taken into account. Here we extend the analysis of Ref. [39] and consider results for (i) the pair approximation that improves on the mean-field analysis and (ii) an agentbased version on one- and two-dimensional regular lattices and complex networks (random and scale free). Interestingly, there is a similarity between the behavior of skeptical agents in our model [39] and the mechanism introduced in the context of a zombie outbreak [40-42], whose analogy with real pandemics was previously explored for didactic purposes $[43,44]$. 
The paper is organized as follows. Section II describes our model and its dynamical rules. Section III summarizes the mean-field results of Ref. [39] and introduces the improved equations within the pair approximation, along with its numerical solutions in both one and two dimensions. We then explore, with numerical simulations (and compare with the results of the previous section), the behavior of the model when spatial correlations are taken into account, both in regular (one and two dimensions; Sec. IV A) and complex networks (random and scale free; Sec. IV B). Finally, the discussion and conclusions are presented in Sec. V.

\section{THE MODEL}

Rumor spreading is modeled here with a set of four possible states (compartments). Some individuals, labeled $Z$, attempt to propagate their opinion by convincing a neighbor who had no previous contact with the information being transmitted. The neighbor is thus susceptible, albeit possibly skeptical (either way, we call it $S$ ). There are two possible outcomes for such pairwise, catalytic interaction between $S$ and $Z$, depending on which agent is modified. First, the $S$ agent may get exposed $(E)$ to the information carried by $Z$ with probability $\beta$ :

$$
S Z \stackrel{\beta}{\longrightarrow} E Z \text {. }
$$

This is similar to the standard susceptible-infected-recovered (SIR) model with exposed agents (SEIR). Another possible result of this interaction, which depends on the degree of (active) skepticism, is the spreader $Z$, after being convinced by a skeptical $S$, gets removed $(R)$ from the population of propagators with probability $\kappa$ :

$$
Z S \stackrel{\kappa}{\longrightarrow} R S .
$$

This latter process, where the rumor propagation can be deflected, is not usually possible in disease propagation. Indeed, in standard SIR models, the spreader does not get modified by interacting with a susceptible. While in the exposed, latent state, the agent may get effectively convinced and, spontaneously, become a spreader with probability $\gamma$ :

$$
E \stackrel{\gamma}{\longrightarrow} Z \text {. }
$$

Otherwise, during the $E$ state, the (skeptical) agent may check the information received and doubt it. As a consequence, it may get removed without any external interaction (passive skepticism), with probability $1-\gamma$ :

$$
E \stackrel{1-\gamma}{\longrightarrow} R \text {. }
$$

By pulling itself out of the spreading process, the agent is no longer capable of changing its state, becoming immune to any further contact with the rumor but also taking no action against the spreaders.

The two possible outcomes discussed above, for the $S Z$ interaction, as a consequence of skeptical and critical thinking, justifies the analogy developed in Ref. [39] between this bidirectional mechanism with the pop-culture concept of zombies and the related apocalypse. Interestingly, several mathematical models have been studied considering such apocalyptic scenarios [40-42]. Our model has an analogous mathematical structure, albeit its interpretation does not deal with the living dead but, instead, with the current crisis where fake news and disinformation may be out of control with profound consequences. Notice that we choose to consider rumor as fake news and critical thinking as a beneficial trait. Nonetheless, it is also possible to have a different interpretation, where the rumor is a correct information and the skeptical are indeed deniers.

\section{MEAN-FIELD AND PAIR APPROXIMATION}

The density of each compartment evolves in time, considering Eqs. (1)-(4), as [39]

$$
\begin{aligned}
& \dot{\rho}_{\mathrm{S}}=-\beta \rho_{\mathrm{SZ}}, \\
& \dot{\rho}_{\mathrm{E}}=\beta \rho_{\mathrm{SZ}}-\rho_{\mathrm{E}}, \\
& \dot{\rho}_{\mathrm{Z}}=\gamma \rho_{\mathrm{E}}-\kappa \rho_{\mathrm{SZ}}, \\
& \dot{\rho}_{\mathrm{R}}=(1-\gamma) \rho_{\mathrm{E}}+\kappa \rho_{\mathrm{SZ}} .
\end{aligned}
$$

There are two integrals of motion, $\rho_{\mathrm{S}}+\rho_{\mathrm{E}}+\rho_{\mathrm{Z}}+\rho_{\mathrm{R}}=1$ and $P \equiv(\beta \gamma-\kappa) \rho_{\mathrm{S}}+\beta \gamma \rho_{\mathrm{E}}+\beta \rho_{\mathrm{z}}$, reducing the number of independent variables. Within the one-site, mean-field (MF) approximation that neglects correlations between different sites, $\rho_{\mathrm{SZ}}=\rho_{\mathrm{S}} \rho_{\mathrm{Z}}$, there are two possible steady states [39], $\left(\rho_{\mathrm{S}}^{*}, \rho_{\mathrm{E}}^{*}, \rho_{\mathrm{Z}}^{*}, \rho_{\mathrm{R}}^{*}\right)$ : either the spreaders are absent in the population, $\left(\rho_{\mathrm{S}}^{*}, 0,0,1-\rho_{\mathrm{S}}^{*}\right) \equiv F_{\mathrm{S}}$, or the susceptibles, $\left(0,0, \rho_{\mathrm{Z}}^{*}, 1-\rho_{\mathrm{Z}}^{*}\right) \equiv F_{\mathrm{Z}}$. The stability of these solutions depends on $\beta, \gamma$, and $\kappa$, as well as on the initial condition for the densities, chosen to consist entirely of susceptible and exposed individuals, $\rho_{\mathrm{S}}^{(0)}+\rho_{\mathrm{E}}^{(0)}=1$. With this choice, $F_{\mathrm{S}}$ is stable whenever $\beta \gamma / \kappa<1$ and $\rho_{\mathrm{s}}^{(0)}>\beta \gamma / \kappa$. Otherwise, $F_{\mathrm{Z}}$ is the stable solution. Thus, at the MF level, there are no stationary states where spreaders and susceptibles coexist in a polarized state. The rumor either spreads over a maximum number of agents $\left(F_{\mathrm{Z}}\right.$ solution) or vanishes, with part of the population remaining unexposed ( $F_{\mathrm{S}}$ solution). However, by including spatial correlations, even at the level of pair approximation (PA) [27], this scenario changes, as shown below.

We extend the analysis of Ref. [39] to include correlations between pairs of sites [27], that are particularly important in models with catalytic reactions. The time evolution of the densities of pairs of strategies now depends on the probability of having triplets:

$$
\begin{aligned}
& \dot{\rho}_{\mathrm{SS}}=-\beta \rho_{\mathrm{SSZ}} \\
& \dot{\rho}_{\mathrm{EE}}=-2 \rho_{\mathrm{EE}}+\beta \rho_{\mathrm{ESZ}} \\
& \dot{\rho}_{\mathrm{ZZ}}=2 \gamma \rho_{\mathrm{EZ}}-\kappa \rho_{\mathrm{SZZ}} \\
& \dot{\rho}_{\mathrm{RR}}=2(1-\gamma) \rho_{\mathrm{ER}}+\kappa \rho_{\mathrm{SZR}} \\
& \dot{\rho}_{\mathrm{SE}}=-\rho_{\mathrm{SE}}+\beta\left(1-\frac{1}{2 d}\right)\left(\rho_{\mathrm{SSZ}}-\rho_{\mathrm{ZSE}}\right) \\
& \dot{\rho}_{\mathrm{SZ}}=\gamma \rho_{\mathrm{SE}}-\frac{\beta+\kappa}{2 d} \rho_{\mathrm{SZ}}-\left(1-\frac{1}{2 d}\right)\left(\beta \rho_{\mathrm{ZSZ}}+\kappa \rho_{\mathrm{SZS}}\right), \\
& \dot{\rho}_{\mathrm{SR}}=(1-\gamma) \rho_{\mathrm{SE}}+\frac{\kappa}{2 d} \rho_{\mathrm{SZ}}-\left(1-\frac{1}{2 d}\right)\left(\beta \rho_{\mathrm{ZSR}}-\kappa \rho_{\mathrm{SZS}}\right) \\
& \dot{\rho}_{\mathrm{ZE}}=\gamma \rho_{\mathrm{EE}}-\rho_{\mathrm{EZ}}+\frac{\beta}{2 d} \rho_{\mathrm{SZ}}+\left(1-\frac{1}{2 d}\right)\left(\beta \rho_{\mathrm{ZSZ}}-\kappa \rho_{\mathrm{SZE}}\right)
\end{aligned}
$$




$$
\begin{aligned}
& \dot{\rho}_{\mathrm{ER}}=(1-\gamma) \rho_{\mathrm{EE}}-\rho_{\mathrm{ER}}+\left(1-\frac{1}{2 d}\right)\left(\beta \rho_{\mathrm{ZSR}}+\kappa \rho_{\mathrm{SZE}}\right), \\
& \dot{\rho}_{\mathrm{ZR}}=\gamma \rho_{\mathrm{ER}}+(1-\gamma) \rho_{\mathrm{EZ}}+\kappa\left(1-\frac{1}{2 d}\right)\left(\rho_{\mathrm{SZZ}}-\rho_{\mathrm{SZR}}\right),
\end{aligned}
$$

where $d=1$ or 2 is the dimensionality of the system and the coefficients account for the different combinations and orientations of the above triplets (see Refs. [45,46]). The number of equations can be further reduced by employing sum rules like $\rho_{\mathrm{S}}=\sum_{x} \rho_{\mathrm{S} x}$ and $\rho_{\mathrm{sz}}=\sum_{x} \rho_{\mathrm{SZ} x}$. With the pair approximation, $\rho_{x y z} \simeq \rho_{x y} \rho_{y z} / \rho_{y}$, the above equations can be closed. All stationary solutions have $\rho_{\mathrm{Sz}}=0$ (i.e., no reacting pairs) and $\rho_{\mathrm{E} x}=0$ because exposed agents eventually decay, $\rho_{\mathrm{E}}=0$. However, since no condition applies to $\rho_{\mathrm{SR}}$ and $\rho_{\mathrm{ZR}}$, besides the mean-field $F_{\mathrm{S}}$ and $F_{\mathrm{Z}}$ solutions [39], susceptibles and spreaders may coexist, but not interact; i.e., their interactions are screened by removed individuals. Differently from the single-site MF approximation [39], we were not able to obtain an analytical expression for the densities at the stationary state. We resort, instead, to numerical methods, integrating Eqs. (6) with a fourth-order Runge-Kutta method. Notice that, in the MF approximation, for a given probability $\gamma$ of an exposed agent becoming a spreader, an increase in the exposition probability $(\beta)$ could be compensated by an equivalent increase in the active skepticism $(\kappa)$; i.e., the results only depend on $\beta \gamma / \kappa$. The PA behavior, on the other hand, does not depend on such a simple ratio. Thus, we consider three particular cases, albeit representative of the overall behavior, in order to compare how spreaders and susceptibles compete. For $\beta \ll \kappa$, the population skepticism toward the rumor is much stronger than the rumor virality, while in the other extreme, $\beta \gg \kappa$, it is the opposite and the rumor outcompetes the skepticism. We also consider the intermediate case, $\beta=\kappa$, where both tendencies are similar.

Figure 1 compares the asymptotic fraction of spreaders and removed individuals as a function of the initial number of susceptibles, $\rho_{\mathrm{s}}^{(0)}$, for the PA in one and two dimensions, with the MF results. We consider a large probability of transforming exposed individuals into spreaders $(\gamma=0.8)$, and several ratios $\beta / \kappa$. For $\rho_{\mathrm{s}}^{(0)}=0$, a fraction $\gamma$ of the all-exposed initial population turns, spontaneously, into spreaders, $\rho_{\mathrm{z}} \simeq \gamma$, the remaining becoming removed, $\rho_{\mathrm{R}} \simeq 1-\gamma$. For small to moderate values of $\beta / \kappa$, spreaders only prevail when the number of susceptibles is small [Figs. 1(a) and 1(b)]. As $\beta$ increases, facilitating the transition $S \rightarrow E$ (and, then, to $Z$ ), there is a change in the concavity of the curve $\rho_{\mathrm{Z}}\left(\rho_{\mathrm{S}}^{(0)}\right)$ : the initial decreasing rate of $\rho_{\mathrm{Z}}$ becomes very small [Figs. 1(a)-1(c)] and the overall fraction of spreaders gets larger, in both one and two dimensions, despite $\rho_{\mathrm{Z}}$ being always a monotonically decreasing function of $\rho_{\mathrm{s}}^{(0)}$.

For $\beta \gg \kappa$ [Fig. 1(c)], the asymptotic fraction of spreaders may be large even for populations initially dominated by susceptibles. In this limit, the information is highly viral and almost no spreader gets removed after being created from the initially exposed individuals. Indeed, in two dimensions, almost independently of $\rho_{\mathrm{s}}^{(0)}$ (except, of course, at $\rho_{\mathrm{s}}^{(0)}=1$ where $\rho_{\mathrm{Z}}=0$ and it is discontinuous), $\rho_{\mathrm{Z}} \simeq \gamma$ and $\rho_{\mathrm{R}} \simeq 1-\gamma$ (then, $\rho_{\mathrm{S}} \simeq 0$ ). This constant behavior, present only in two dimensions, is remarkable since the more susceptibles (that

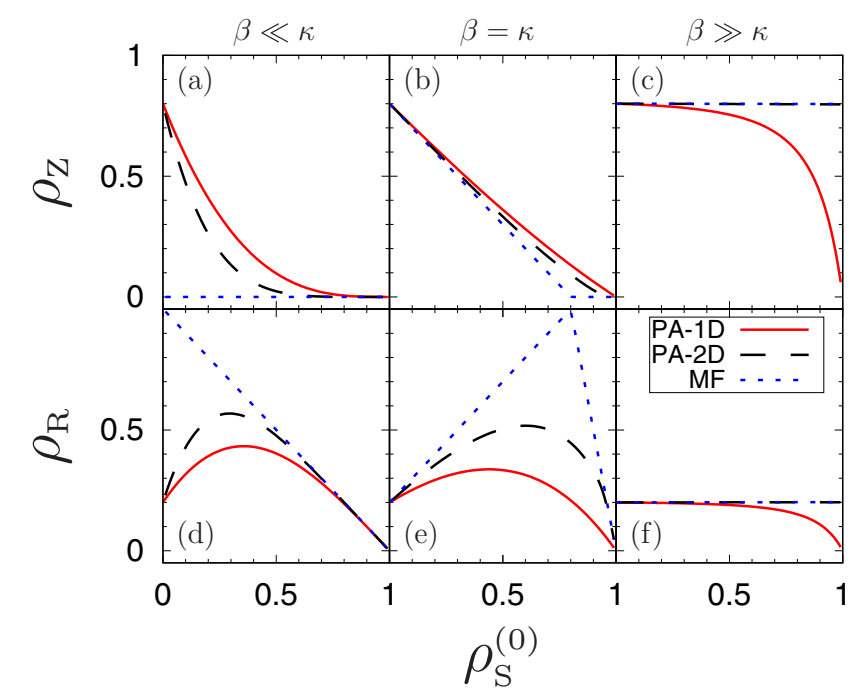

FIG. 1. Asymptotic density of spreaders, $\rho_{\mathrm{Z}}$ (top row), and removed, $\rho_{\mathrm{R}}$ (bottom row), as a function of the initial fraction of susceptibles, $\rho_{\mathrm{S}}^{(0)}=1-\rho_{\mathrm{E}}^{(0)}$. The results allow the comparison between the different levels of approximation: mean-field approximation (dotted line) and the pair approximation for one (solid line) and two dimensions (dashed line). The relation between $\beta$ and $\kappa$ is indicated in each column, from left to right: $\beta \ll \kappa\left(\beta=10^{-3}\right.$ and $\left.\kappa=0.8\right)$, $\beta=\kappa=0.1$ and $\beta \gg \kappa\left(\beta=0.8\right.$ and $\left.\kappa=10^{-3}\right)$. Notice that in panels (c) and (f), the MF and the PA for two dimensions coincide. In all cases, $\gamma=0.8$.

may be skeptics) are present in the initial state, the more resistance is to be expected, helping to prevent the increase of spreaders, as observed in all other cases. There is, however, a qualitative difference with the one-dimensional (1D) case where there is an appreciable reduction in both $\rho_{\mathrm{Z}}$ and $\rho_{\mathrm{R}}$ when $\rho_{\mathrm{S}}^{(0)}$ increases (i.e., $\rho_{\mathrm{S}}>0$ ). This strong dependence on the dimension has its origin in the different coordination and the possibility of having multiple paths connecting two sites in two dimensions. In one dimension, on the contrary, groups of susceptible agents may easily become isolated once they are located between two removed individuals, preventing their exposition to spreaders and halting any further transformation. As a consequence, $\rho_{\mathrm{S}}$ remains finite and increases with $\rho_{\mathrm{S}}^{(0)}$, while $\rho_{\mathrm{Z}}$ decreases.

Figure 1 also shows (bottom row) the population of removed individuals, $\rho_{\mathrm{R}}$, as a consequence of the skepticism among the population. Their number is relevant when designing a strategy to prevent rumor spreading. Up to the level of the pair approximation, in spite of the rumor never completely vanishing, some control over its global spreading can be obtained by properly tuning either the initial conditions or the parameters of the model. Specifically, despite our results never showing an all-removed scenario, there usually is a maximum value of $\rho_{\mathrm{R}}\left(\rho_{\mathrm{S}}^{(0)}\right)$ that optimizes the resistance against the rumor [Figs. 1(d) and 1(e)], for $\beta \leqslant \kappa$. Nonetheless, for $\beta \gg \kappa$ [Fig. 1(f)], the maximum $\rho_{\mathrm{R}}$ always occurs at $\rho_{\mathrm{S}}^{(0)}=0$. On the other hand, as a function of $\gamma$ (not shown), all cases present a maximum.

The main observed difference between the pair and meanfield approximations is related to the possibility of spreaders, 
susceptibles, and removed agents coexisting. Within the PA this is, in fact, the only solution found, even for the extremes $\beta \ll \kappa$ and $\beta \gg \kappa$, where the population of spreaders and susceptibles, respectively, becomes very small, but does not disappear. Indeed, a more extensive analysis would be necessary before ruling out solutions like $F_{\mathrm{S}}$ or $F_{\mathrm{Z}}$ that seem to appear, rather trivially, for $\rho_{\mathrm{s}}^{(0)}=0$ and 1 . However, these states also occur when $\beta=1, \kappa=0$ with $\gamma \approx 0$, a particular situation where the final population is formed only by susceptibles and removed individuals $\left(F_{\mathrm{S}}\right)$, or if $\beta=0, \kappa=1$ with $\gamma \approx 1$, when the steady state is $F_{\mathrm{Z}}$. Both cases are independent of the initial population of susceptibles, $\rho_{\mathrm{s}}^{(0)}$.

\section{SPATIALLY DISTRIBUTED SYSTEMS}

In the previous section we improved on the MF analysis of Ref. [39] by taking into account correlations on the level of couples of sites (the PA). These results should be compared with the simulations on spatial lattices, exploring the effects of these correlations on the steady state. We consider three different networks on which $N$ sites are arranged: random, complex (scale-free), and $d$-dimensional regular lattices with side $L\left(N=L^{d}\right)$ and periodic boundary conditions. In each site there is a single agent that, initially, is set to be either $E$ or $S$. Along the dynamics, a site is chosen at random and updated accordingly with Eqs. (1)-(4): an $E$ decays into $Z$ or $R$ with probability $\gamma$ or $1-\gamma$, respectively, irrespective of the neighborhood, while if it is a $Z$ or an $S$, a neighbor is also randomly chosen and, if appropriate, either Eq. (1) or (2) is used with the corresponding probability. The asymptotic densities are measured for different system sizes and averages are taken over 100 to 200 samples. When the size is not explicitly mentioned, the results were extrapolated to $L \rightarrow \infty$ using a simple scaling, $\rho_{x}^{(\infty)}-\rho_{x}^{(L)} \sim L^{-1}$, which fits quite well the numerical data.

\section{A. Regular lattices \\ 1. One-dimensional case}

We consider linear systems with periodic boundary conditions and sizes ranging from $L=10^{5}$ to $10^{6}$. The behavior of $\rho_{\mathrm{z}}$, shown in Fig. 2 for several values of the parameters, is qualitatively similar to the PA. Spreaders and susceptibles coexist by forming isolated islands where the intermediate removed individuals prevent any interaction. There are, however, some differences between both cases. In spatial systems, spreaders are the prevalent species in a slightly smaller region of the $\left(\rho_{\mathrm{s}}^{(0)}, \gamma\right)$ plane. Moreover, as can be seen in Fig. 3(a), the PA usually underestimates $\rho_{\mathrm{z}}$ (see, for example, the curves for $\beta \ll \kappa$ and $\beta=\kappa$ ). However, for $\beta \gg \kappa, \rho_{\mathrm{z}}$ develops a small plateau (observed only in the spatial 1D case), before which the pair approximation gives a larger estimate for $\rho_{\mathrm{z}}$. Although there is a reasonable quantitative agreement between the simulation and the PA for $\rho_{\mathrm{Z}}$, the comparison with $\rho_{\mathrm{R}}$ is not as good, as can be seen in Fig. 3(c). Another interesting feature is that usually the partition between spreaders and susceptibles is asymmetric, even for $\beta=\kappa$, with the maximum symmetry (measured, for example, by the product $\rho_{\mathrm{Z}} \rho_{\mathrm{S}}$ ) occurring for $\beta \gg \kappa$ in a domain where $\gamma \approx 1$ and $\rho_{\mathrm{s}}^{(0)} \approx 1$.

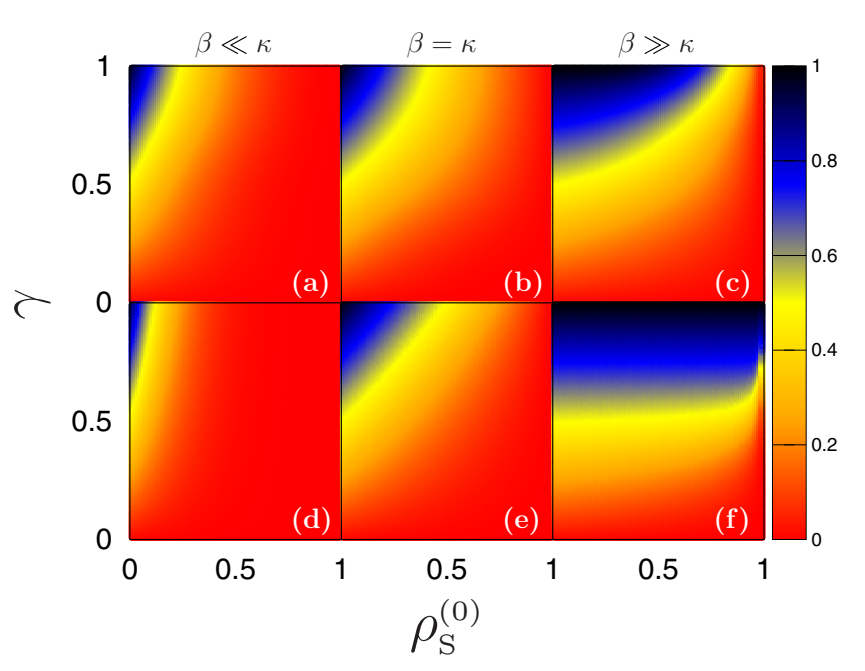

FIG. 2. Asymptotic density of spreaders $\rho_{\mathrm{Z}}$ for spatial systems in one dimension $\left(L=5 \times 10^{5}\right.$, top row) and two dimensions $(L=$ 500 , bottom row). As indicated on the top of each column, from left to right: $\beta \ll \kappa, \beta=\kappa$, and $\beta \gg \kappa$.

\section{Two-dimensional case}

In two dimensions, we consider square lattices with $L^{2}$ sites $\left(L=10^{2}-10^{3}\right)$ and periodic boundary conditions along both directions. Differently from the 1D case, the agreement between the simulation and the PA is excellent, suggesting that the reactions induce strong pair correlations that dominate the stationary state. This short-range coupling is related to the compact clusters of spreaders or susceptibles, whose interactions are inhibited by intermediate removed individuals. These $R$ agents, since they no longer interact, prevent any further evolution of the system. Models that are similar, but that do

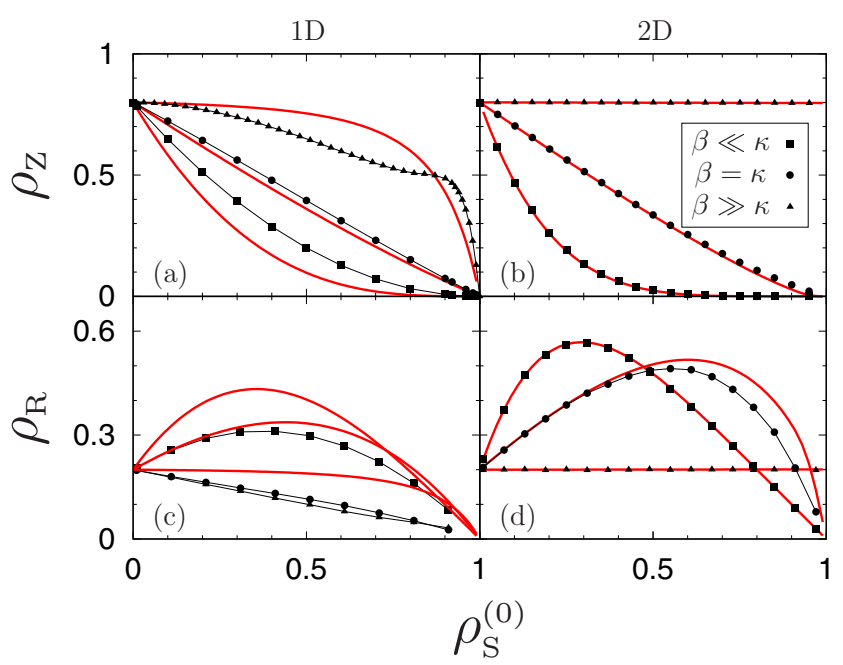

FIG. 3. Asymptotic density of spreaders, $\rho_{\mathrm{Z}}$ (top), and removed (bottom) as a function of $\rho_{\mathrm{S}}^{(0)}$ for both one dimension (left) and two dimensions (right). The solid lines are the results from the PA with $\gamma=0.8$, while the points are extrapolations, for $L \rightarrow \infty$, of the corresponding simulations. Notice the excellent agreement in the two-dimensional (2D) case. 


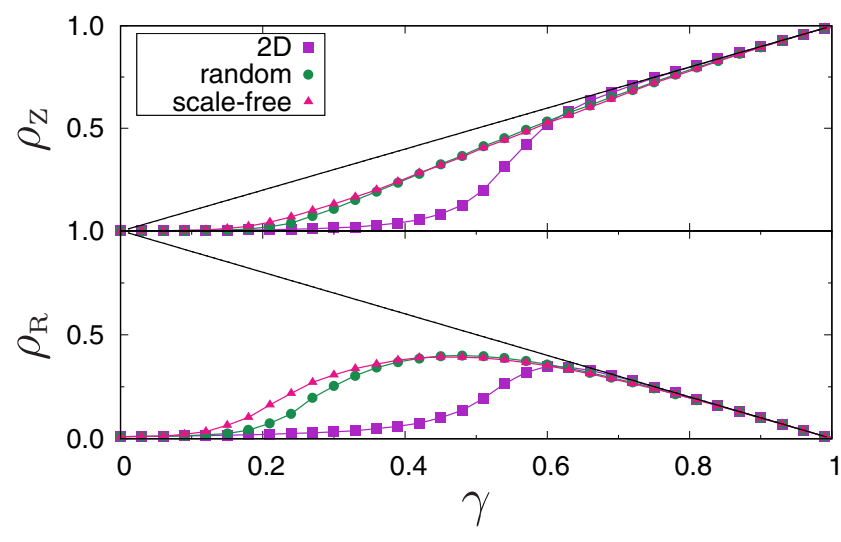

FIG. 4. Asymptotic fraction of spreaders $\left(\rho_{\mathrm{Z}}\right.$, top panel) and removed agents $\left(\rho_{\mathrm{R}}\right.$, bottom panel) as a function of $\gamma$ with $N=10^{4}$ for regular, random, and scale-free networks, along with the meanfield result (straight line). We consider $\beta \gg \kappa$ and $\rho_{\mathrm{S}}^{(0)}=0.99$.

not sustain an equilibrium coexistence of three species, do not show such agreement between the PA and simulations.

The density of removed individuals measured in the simulation agrees well with the pair approximation predictions [Fig. 3(d)], although some deviation can be observed for $\beta=\kappa$ and large $\rho_{\mathrm{s}}^{(0)}$. Relevant information may be also obtained from the position and height of the peak of $\rho_{\mathrm{R}}$ which are, respectively, an increasing and decreasing function of $\gamma$ (not shown). Thus, for a given virality, there is an optimal fraction of susceptibles/skeptical agents that, if present in the beginning of the propagation process, will counteract by producing the largest fraction of removed individuals. When $\gamma$ is large, exposed individuals are easily converted into spreaders and, because this maximum value increases with $\gamma$, the initial concentration of susceptibles has to be large enough to enforce a maximum removal due to skepticism. Notice that even in the limit $\gamma \rightarrow 1$ (high virality), the amount of these removed agents is still large (not shown). Nonetheless, the effectiveness, i.e., the actual fraction of removed agents at the maximum, decreases with $\gamma$.

\section{B. Scale-free and random networks}

Social networks are neither fully connected nor regular, being indeed better described by complex networks. We thus extend the previous analysis to include random and scale-free networks, both built using the Krapivsky-Redner algorithm [47]. Figure 4 presents the behavior of $\rho_{\mathrm{Z}}$ and $\rho_{\mathrm{R}}$, as a function of $\gamma$, in the region $\beta \gg \kappa$ for the regular, random, and scale-free networks, along with the MF approximation results (straight lines). In this regime of high rumor virality, all densities are much more sensitive to $\gamma$ than to $\rho_{\mathrm{s}}^{(0)}$. Except for large $\gamma$ where MF nicely agrees with the simulations (and all three networks behave the same), both spreaders (top panel) and removed (bottom panel) are overestimated in the MF approximation while susceptibles are underestimated.

For the model we consider here, the overall qualitative behavior observed on these three structures is similar. Quantitatively, although the difference between the random and the scale-free networks is small in the whole interval, they strongly differ from the square lattice where the amount of removed agents is much smaller. This robustness regarding the topology of connections seems to have a common origin on the strong pair correlations, as indicated by the good agreement with the pair-approximation results. Moreover, it is related to the permanent screening role of the removed, preventing further interactions between susceptibles and spreaders and, consequently, halting the dynamics (exposed agents have a similar, albeit transient role). In order to be effective, any strategy cluster must be surrounded by removed individuals. This is the case of the square lattice since the nearest-neighbor groups with either $S$ or $Z$ agents are more compact and, because of that, need a smaller number of removed to fully cover their surface. Once long-range connections are present, as in the random and scale-free networks, such a surface becomes larger and more diffuse, and a larger number of removed individuals is necessary to cover it. In the MF limit, where the network is fully connected, it is no longer possible to prevent the interactions between spreaders and susceptibles and they cannot coexist. Indeed, the asymptotic state becomes a mixture of removed and either susceptibles $\left(F_{\mathrm{S}}\right)$ or spreaders $\left(F_{\mathrm{Z}}\right)$.

These results hint to a robust effect, where the sole nature of the interactions, either short or long range, determines whether the fraction of removed agents is enough to stabilize the coexistence state. While long-range interactions can initially lead to a wider spreading of the rumor, at the same time the $S Z$ interactions produce a larger fraction of removed individuals in the final state. This is observed (Fig. 4) both in random and complex networks, where despite the distribution of connectivity being different, the results are much alike. The local pattern of connections in these lattices differs from the square lattice, making the protective effect of removed agents less efficient. Indeed, on a square lattice, because connected susceptibles form a compact group whose surface is much smaller, fewer removed individuals are necessary to cover such a surface, protecting the susceptibles. The more longrange interactions are present, the more difficult it becomes to completely surround the group. Because of this, random and complex networks are more easily invaded by spreaders, leading to a larger number of removed agents. The fully connected limit corresponding to MF is an extreme case where such protection is impossible, preventing any coexistence between spreaders and susceptibles. On the square lattice, because the number of removed and spreaders is smaller than in complex networks, there are more susceptibles. This is a general feature, observed on most of the parameter space.

There are, in this model, two possible ways to control the propagation of a rumor and, consequently, have a small asymptotic fraction of spreaders, $\rho_{\mathrm{Z}} \rightarrow 0$. A direct mechanism occurs when the passive skepticism within the population is large $(\gamma$ is small and almost no exposed individual becomes a spreader). Nonetheless, typical populations do not have a small $\gamma$. Usual, intermediate values of $\gamma$ allow an indirect mechanism with a larger number of removed agents that protect the population of susceptibles, isolating them from the spreaders and allowing a stable coexistence between the two populations.

Figure 5 provides a geometric perspective on the time evolution for the square lattice in the region $\beta \gg \kappa$ and three different values of $\gamma$ around the peak at $\gamma \simeq 0.6$ (see Fig. 4). 

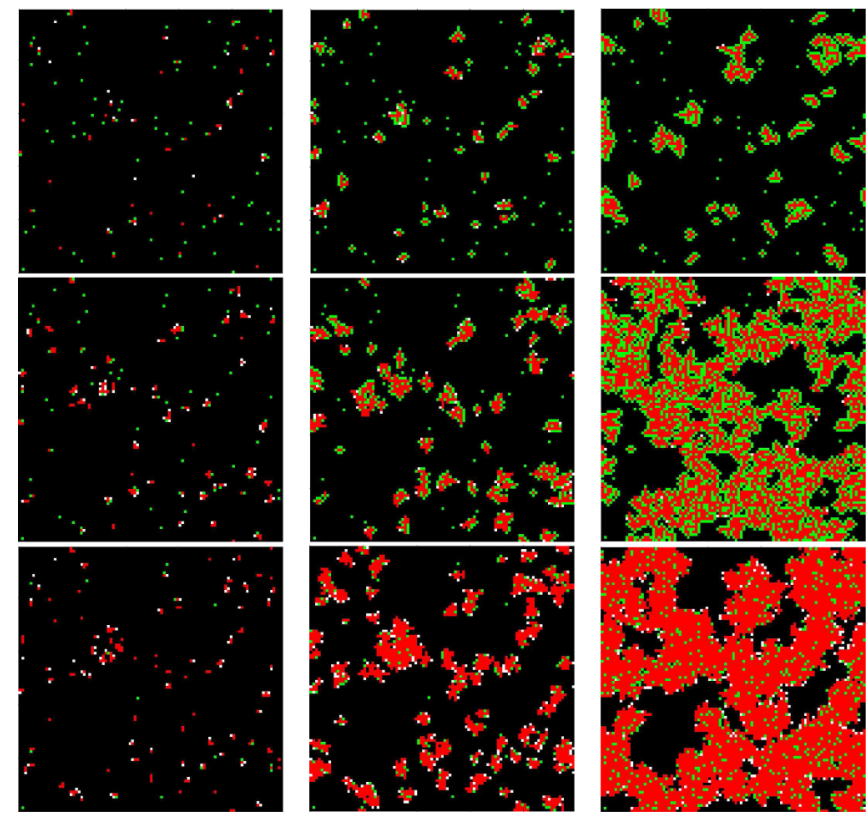

FIG. 5. Snapshots for the square lattice with $\gamma=0.4,0.6$, and 0.9 (from top to bottom, corresponding to the region before, at, and after the peak of $\rho_{\mathrm{R}}$ at $\gamma \simeq 0.6$ in Fig. 4) at three different times (increasing from left to right). The color code is black $(S)$, white $(E)$, red $(Z)$, and green $(R)$. Notice that, in the third row, the final snapshot is not the asymptotic state of the population (spreaders will dominate and only a few susceptibles survive, isolated by removed agents).

The screening effect of the removed agents strongly changes the evolution of the system. For a value of $\gamma$ to the left of the peak (top row), spreaders remain confined in small groups, despite the large virality $(\beta \gg \kappa)$, with removed individuals on the surface. In this case, the number of removed agents necessary to cover the total surface is small. On the other hand, to the right of the peak (larger $\gamma$, bottom row), the number of removed is small and, as a consequence, not enough to prevent spreaders from invading the whole population. The infection rate is so high that there are almost no susceptibles in the final state. For intermediate values of $\gamma$, close to the peak (middle row) the number of removed individuals in the central panel is not enough to constrain the whole population of spreaders and a small fraction keeps infecting and removing agents from the population. We now further explore the conditions that induce a large fraction $\rho_{\mathrm{R}}$ in the parameter space.

While MF predicts that $\rho_{\mathrm{R}}=1-\gamma$, for the three structured topologies considered here, the number of removed individuals presents a nonlinear behavior as a function of $\gamma$ : there is a minimum value of $\rho_{\mathrm{s}}^{(0)}$ above which there is a peak in $\rho_{\mathrm{R}}$ when $\beta \gg \kappa$. As shown in Fig. 6, for decreasing values of $\rho_{\mathrm{s}}^{(0)}$, the peak moves to the left, widens, and increases in height, eventually arriving at $\gamma=0$ for a finite $\rho_{\mathrm{s}}^{(0)}$. Obviously, if $\rho_{\mathrm{s}}^{(0)}=0$, the exposed individuals in the initial state become removed with probability $1-\gamma$, the same result as in the MF approach (the upper bound, straight line in Fig. 6). Notice that all curves merge with this MF result for large $\gamma$. Even if $\rho_{\mathrm{s}}^{(0)}>0$, most of the exposed agents become spreaders that, in turn (in the limit considered here, of large $\gamma$ and $\beta \gg \kappa$ ), help remove the remaining susceptibles. Eventually, removed and spreaders

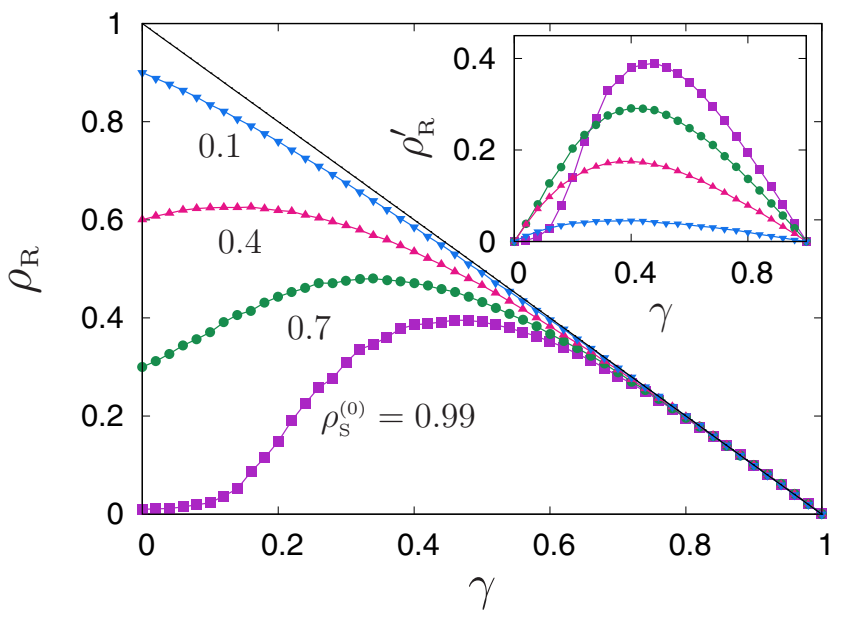

FIG. 6. Asymptotic fraction of removed agents $\rho_{\mathrm{R}}$ as a function of $\gamma$ for several values of $\rho_{\mathrm{S}}^{(0)}$ in the scale-free network with $\beta \gg \kappa$. The straight line is the MF result $\rho_{\mathrm{R}}=1-\gamma$. Inset: The corresponding asymptotic fraction of secondary removed individuals after discounting the contribution from the initial state, $\rho_{\mathrm{R}}^{\prime}=\rho_{\mathrm{R}}-$ $\rho_{\mathrm{E}}^{(0)}(1-\gamma)$.

seem to be the only agents remaining (whether susceptibles actually disappear or a very small fraction remains depends on further, more detailed simulations). In our model, there are two main mechanisms that remove agents: the reaction $Z S \rightarrow$ $R S$ with a rate $\kappa$, and the spontaneous decay $E \rightarrow R$ with rate $1-\gamma$. At the $\rho_{\mathrm{R}}$ peak there are contributions from both processes. The effect of the leading mechanism in each side of the peak can be seen in the snapshots of Fig. 5. Thus, the peak separates the regimes where spreaders still have contact with susceptibles, and the point where most of the latter only survive inside isolated islands surrounded by removed. To quantify the removed individuals that do not originate from the exposed agents already present in the initial state, we show $\rho_{\mathrm{R}}^{\prime}=\rho_{\mathrm{R}}-\rho_{\mathrm{E}}^{(0)}(1-\gamma)$ in the inset of Fig. 6 as a function of $\gamma$. Notice that the role played by $\rho_{\mathrm{S}}^{(0)}$ is reversed when considering only the agents whose removal was inherent to the population dynamics, not those in the initial state: a larger peak is observed when more susceptibles are present in the initial state. Indeed, in the main panel of Fig. 6, the case with the smaller peak becomes the largest in the inset. Moreover, $\rho_{\mathrm{R}}^{\prime}$ presents a peak for all values of $\rho_{\mathrm{S}}^{(0)}$, even those for which $\rho_{\mathrm{R}}$ is monotonic. After the peak, the linear behavior of $\rho_{\mathrm{R}}^{\prime}$ is a direct consequence of the collapsed behavior seen in the corresponding region of Fig. 4: in that region, $\rho_{\mathrm{R}} \simeq 1-\gamma$ and $\rho_{\mathrm{R}}^{\prime} \simeq 1-\gamma-\rho_{\mathrm{E}}^{(0)}(1-\gamma)=\rho_{\mathrm{s}}^{(0)}(1-\gamma)$. Indeed, by plotting $\rho_{\mathrm{R}}^{\prime} / \rho_{\mathrm{S}}^{(0)}$, a similar collapse is obtained (not shown). This can be seen as a decoupling of the effect of $\gamma$ on the final population of $\rho_{\mathrm{R}}$; i.e., the secondary removed individuals will depend on $\gamma$ but not in a simple way.

\section{CONCLUSIONS}

We considered a simple rumor-spreading model that includes agent-level skepticism [39]. An individual that had contact with a rumor may become removed (disinterested) from the propagation process following two different routes, 
both driven by skeptical inquiry. The first, if it is a spreader, is by getting in contact with a skeptical susceptible. This is a direct, active mechanism based on persuasive argumentation. The second, if it has only been exposed but not yet turned into a spreader, is by discarding the rumor (passive mechanism) due, for instance, to fact checking. Prior work [39] studied this model within the mean-field (MF) approximation. Here we extend those results by including correlations among neighboring pairs of agents (pair approximation), and compare with agent-based simulations in different geometries (one- and two-dimensional lattices and random and scalefree networks), showing that the two-site approximation well describes the simulation results. Neighborhood, in this case, refers to social contacts as spatially close individuals do not always exchange information.

Removed sites have an important function acting as barriers for rumor spreading when spatial effects are taken into account. In the asymptotic, stationary state, these removed agents may coexist with susceptibles and spreaders. The mean-field approach, being equivalent to a fully connected system, does not provide such possibility, and either $S$ or $Z$ get extinct (preliminary results show that when including diffusion, as the effective range of interactions increase, the lattice results approach those of the MF approach). Notice also that although these MF solutions were not observed in the simulations, more extensive simulations should be performed in order to completely rule this possibility out. In order to be stable, the coexisting susceptibles and spreaders must be spatially isolated, their interactions hindered by the surrounding removed individuals. Square lattices may present a larger fraction of susceptible individuals as compared to complex networks for a given set of parameters. The latter has, instead, more removed agents (a relevant parameter as it corresponds to those that lost interest in spreading the rumor). The reason is that susceptibles form more compact groups in regular lattices, thus needing a smaller number of protective removed agents on their surface. The long-range interactions present in the complex networks increase the probability of $S Z$ encounters, and a correspondent larger number of removed individuals. This indeed is the mechanism allowing social networks to easily spread information. Interestingly, collective, long-ranged conveyors of information like magazines, radio, and television, present before the advent of the Internet, did not have such capability because of the traditional fact checking that most of the media enforces. When individuals start to propagate their own beliefs and opinions, because skeptical inquiry and critical thinking are not, yet, widely held capabilities, rumor contention becomes a very difficult task to which, presently, there is no efficient solution available.

Some spatial contention may be obtained by attaining a sufficient degree of herd immunity. A similar result, for disease spreading, is traditionally obtained through vaccination programs. Although not all individuals may have their skeptical immunological system fully developed, pseudoscience and fake-news propagation may be halted by protecting vulnerable groups through a large and well-distributed population of sufficiently educated people. It is important to stress again that, in the stationary state, spreaders and removed individuals may coexist. As indicated by experimental observations, while new conspiracy theories and pseudoscience are constantly invented and disseminated, they rarely replace entirely the previous ones, not even when actively targeted by skeptical individuals. In these situations, while completely eliminating fake-news spreaders may not be feasible, achieving the optimal fraction of individuals not interested in rumors, i.e., removing then from the propagation process, can be a more realistic goal. We observe that intermediate values of $\gamma$ (the rate of spontaneous transitions between exposed and spreader) seem to generate a larger fraction of removed individuals when the rumor is highly viral $(\beta \gg \kappa)$. Interestingly, after correcting the final fraction of removed to exclude the initially exposed individuals that will unavoidably be removed, we observe that this effect is even more pronounced when the initial number of exposed individuals is small.

From the perspective of fake-news spreading, this can be understood as a protective effect that an initial exposure can cause in a (sufficiently) skeptical population. If there are no passively skeptical individuals (high $\gamma$ ), the rumor will, evidently, dominate the population, with a high final $\rho_{\mathrm{z}}$. But if the passive skepticism is too high (low $\gamma$ ), the rumor will be quickly trapped inside a small island of removed (disinterested) individuals. While this can create a large final fraction of susceptible agents, it also means that most individuals never had contact with the rumor, leaving the final population still susceptible to future rumors. Finally, an intermediate value of $\gamma$ near this point can make the rumor spread through the population in a controlled way, creating more removed individuals than any other case. While this is not a perfect scenario, a population with the highest number of disinterested individuals will be more resilient to rumor spreading.

Reference [39] emphasized that the mechanism used by skeptical agents to individually counteract the propagation of rumors is similar to the pop-culture scenarios for a zombie outbreak (both rumors and zombies may be directly eliminated by susceptibles agents). Such a picture has been previously used to communicate the science of real epidemics and the advantages of preparedness [43], while also motivating some theoretical studies [40-42]. On a global level, the current dissemination of pseudoscience and disinformation, along with the widespread phenomenon of authorities discrediting scientists (e.g., the recent climate emergency and coronavirus epidemic), everything facilitated by our technology, is the equivalent of a zombie apocalypse. Thus, this class of models remains interesting as we may get insight on how skeptics should act to stop rumor spreading. In particular, a better understanding of how rumor propagates and how effective can be an effort to resist or even to convince people may help to devise intervention strategies focused on specific individuals in a similar way as crime and Internet hate control.

\section{ACKNOWLEDGMENTS}

W.G.D. acknowledges the hospitality of the IF-UFRGS during his stay where part of this work was done. Work was partially supported by the Brazilian agencies FAPERGS (Grant No. 19/2551-0000555-5), FAPERJ, CNPq (Process No. 428653/2018-9), and CAPES (Finance code 001). 
[1] A. Bessi, M. Coletto, G. A. Davidescu, A. Scala, G. Caldarelli, and W. Quattrociocchi, PLoS One 10, e0118093 (2015).

[2] M. Del Vicario, A. Bessi, F. Zollo, F. Petroni, A. Scala, G. Caldarelli, H. E. Stanley, and W. Quattrociocchi, Proc. Natl. Acad. Sci. USA 113, 554 (2016).

[3] C. O'Connor and J. O. Weatherall, Eur. J. Philos. Sci. 8, 855 (2018).

[4] F. Baumann, P. Lorenz-Spreen, I. M. Sokolov, and M. Starnini, Phys. Rev. Lett. 124, 048301 (2020).

[5] S. Galam, Int. J. Mod. Phys. C 19, 409 (2008).

[6] C. Castellano, S. Fortunato, and V. Loreto, Rev. Mod. Phys. 81, 591 (2009).

[7] T. Britton, Math. Biosci. 225, 24 (2010).

[8] R. Pastor-Satorras, C. Castellano, P. Van Mieghem, and A. Vespignani, Rev. Mod. Phys. 87, 925 (2015).

[9] G.-Q. Sun, M. Jusup, Z. Jin, Y. Wang, and Z. Wang, Phys. Life Rev. 19, 43 (2016).

[10] G. F. de Arruda, F. A. Rodrigues, and Y. Moreno, Phys. Rep. 756, 1 (2018).

[11] R. Morton and K. H. Wickwire, Adv. Appl. Probab. 6, 622 (1974).

[12] M. A. Strassburg, Am. J. Infect. Control 10, 53 (1982).

[13] S. Funk, M. Salathé, and V. A. A. Jansen, J. R. Soc. Interface 7, 1247 (2010).

[14] D. L. Smith, K. E. Battle, S. I. Hay, C. M. Barker, T. W. Scott, and F. E. McKenzie, PLoS Pathogens 8, e1002588 (2012).

[15] Z. Wang, C. T. Bauch, S. Bhattacharyya, A. d'Onofrio, P. Manfredi, M. Perc, N. Perra, M. Salathé, and D. Zhao, Phys. Rep. 664, 1 (2016).

[16] L. Bolzoni, E. Bonacini, R. D. Marca, and M. Groppi, Math. Biosci. 315, 108232 (2019).

[17] G. S. Costa and S. C. Ferreira, Phys. Rev. E 101, 022311 (2020).

[18] H.-X. Yang, M. Tang, and Z. Wang, Physica A 490, 347 (2018).

[19] P. Bittihn and R. Golestanian, arXiv:2003.08784.

[20] F. Kato, K.-I. Tainaka, S. Sone, S. Morita, H. Iida, and J. Yoshimura, Sci. Rep. 1, 10 (2011).

[21] A. Dobay, G. E. Gall, D. J. Rankin, and H. C. Bagheri, J. Theor. Biol. 317, 348 (2013).

[22] T. Hasegawa and K. Nemoto, Phys. Rev. E 96, 022311 (2017).

[23] N. F. Johnson, R. Leahy, N. J. Restrepo, N. Velasquez, M. Zheng, P. Manrique, P. Devkota, and S. Wuchty, Nature (London) 573, 261 (2019).

[24] B. R. da Cunha and S. Gonçalves, Appl. Netw. Sci. 3, 36 (2018).

[25] S. Galam and M. A. Javarone, PLoS One 11, e0155407 (2016).
[26] M. Santoprete, Appl. Math. Comput. 358, 314 (2019).

[27] J. Marro and R. Dickman, Nonequilibrium Phase Transitions in Lattice Models (Cambridge University Press, Cambridge, UK, 1999).

[28] W. Goffman and V. A. Newill, Nature (London) 204, 225 (1964).

[29] D. J. Daley and D. G. Kendall, Nature (London) 204, 1118 (1964).

[30] D. J. Daley and D. G. Kendall, IMA J. Appl. Math. 1, 42 (1965).

[31] W. Goffman and V. A. Newill, Proc. R. Soc. A 298, 316 (1967).

[32] D. P. Maki and M. Thompson, Mathematical Models and Applications (Prentice-Hall, Englewood Cliffs, NJ, 1973).

[33] W. O. Kermack and A. G. McKendrick, Proc. R. Soc. A 115, 700 (1927).

[34] F. Brauer, Infect. Dis. Mod. 2, 113 (2017).

[35] J. L. Aron and I. B. Schwartz, J. Theor. Biol. 110, 665 (1984).

[36] L.-L. Xia, G.-P. Jiang, B. Song, and Y. R. Song, Physica A 437, 295 (2015).

[37] M. Tambuscio, D. F. M. Oliveira, G. L. Ciampaglia, and G. Ruffo, J. Comput. Soc. Sci. 1, 261 (2018).

[38] A. N. Zehmakan and S. Galam, arXiv:1905.06894.

[39] M. A. Amaral and J. J. Arenzon, Europhys. Lett. 124, 18007 (2018).

[40] P. Munz, I. Hudea, J. Imad, and R. J. Smith, in Infectious Disease Modelling Research Progress, edited by J. M. Tchuenche and C. Chiyaka (Nova Science Publishers, New York, 2009), Chap. 4, pp. 133-150.

[41] A. A. Alemi, M. Bierbaum, C. R. Myers, and J. P. Sethna, Phys. Rev. E 92, 052801 (2015).

[42] R. Hochreiter and C. Waldhauser, Soft Comput. 24, 591 (2020).

[43] U.S. Department of Health and Human Services, Centers for Disease Control and Prevention, http://www.cdc.gov/phpr/ zombies.

[44] J. Verran, M. Crossley, K. Carolan, N. Jacobs, and M. Amos, J. Biol. Educ. 48, 98 (2014).

[45] M. van Baalen, in The Geometry of Ecological Interactions: Simplifying Spatial Complexity, edited by U. Dieckmann, R. Law, and J. A. J. Metz (Cambridge University Press, Cambridge, UK, 2000), pp. 359-387.

[46] G. Szabó and G. Fáth, Phys. Rep. 446, 97 (2007).

[47] P. L. Krapivsky and S. Redner, Phys. Rev. E 63, 066123 (2001). 